Теорія Ймовір. та Матем. Статист. Вип. 74, 2006
Theor. Probability and Math. Statist.

No. 74, 2007, Pages 171-179

S 0094-9000(07)00706-5

Article electronically published on July 5, 2007

\title{
A PROBLEM OF INTERPOLATION OF A HOMOGENEOUS AND ISOTROPIC RANDOM FIELD
}

UDC 519.21

\author{
N. SEMENOVS'KA
}

\begin{abstract}
A solution to the interpolation problem for the value of a homogeneous and isotropic random field at an arbitrary point inside an $n$-dimensional sphere after observations on a finite set of points on the sphere is found. The asymptotic behavior of the interpolation error as the number of points increases is studied. Recommendations on where the observation points should be placed on the sphere are given.
\end{abstract}

\section{INTRODUCTION}

In paper [1, the problem of interpolation of a random field at the center of a circle on the plane was considered. Here, we extend these results: we study a multidimensional space $(n>2)$ and, moreover, we consider interpolation at an arbitrary point inside an $n$-dimensional sphere. We employ notation introduced in the above-mentioned paper.

\section{Homogeneous AND ISOTROPIC RANDOM FIELDS ON $\mathbb{R}^{n}$}

Let us recall some facts from spectral theory of random fields. Assume that $\xi(x)$, $x \in \mathbb{R}^{n}$, is a homogeneous and isotropic random field on a Euclidean space $\mathbb{R}^{n}$, that is, $\mathrm{E} \xi(x)=$ const (for simplicity, we suppose that $\mathrm{E} \xi(x)=0$ without any loss of generality), $\mathrm{E}|\xi(x)|^{2}<\infty$, and the correlation function

$$
\varphi(|x-y|)=\mathrm{E} \xi(x) \overline{\xi(y)}
$$

depends on the distance $|x-y|$ between the points $x$ and $y$ only.

Assume that $(r, \bar{\varphi})=\left(r, \varphi_{1}, \ldots, \varphi_{n-1}\right)$ are spherical coordinates of the point $x, S_{m}^{l}(\bar{\varphi})$ are orthonormal spherical harmonics of order $m$, and

$$
h(m, n)=(2 m+n-2)(m+n-3) ! /((n-2) ! m !)
$$

is the total number of these harmonics. Suppose that $\xi(x), x \in \mathbb{R}^{n}$, is a mean square continuous random field. Then, by Theorem 1 in [2, I, §1], the following spectral decomposition holds for the field $\xi(x), x \in \mathbb{R}^{n}$ :

$$
\xi(x)=c_{n} \sum_{m \geq 0} \sum_{l=1}^{h(m, n)} S_{m}^{l}(\bar{\varphi}) \int_{0}^{\infty} \frac{J_{m+(n-2) / 2}(\lambda r)}{(\lambda r)^{(n-2) / 2}} Z_{m}^{l}(d \lambda),
$$

2000 Mathematics Subject Classification. Primary 60J60.

Key words and phrases. Homogeneous and isotropic random fields, interpolation, approximation, limit of the error of approximation. 
where $Z_{m}^{l}(\cdot)$ is a sequence of uncorrelated random measures defined on Borel sets in $(0,+\infty)$ such that their spectral measure is $\Phi$ and

$$
\begin{gathered}
\mathrm{E} Z_{m}^{l}(S)=0 \\
\mathrm{E} Z_{m}^{l}\left(S_{1}\right) \overline{Z_{p}^{q}\left(S_{2}\right)}=\delta_{m}^{p} \delta_{l}^{q} \Phi\left(S_{1} \cap S_{2}\right)
\end{gathered}
$$

for any Borel sets $S, S_{1}$, and $S_{2}$ belonging to $(0,+\infty)$. Here, $c_{n}^{2}=2^{n-1} \Gamma(n / 2) \pi^{n / 2}$.

Put

$$
\zeta_{m}^{l}(r)=\int_{0}^{\infty} \frac{J_{m+(n-2) / 2}(\lambda r)}{(\lambda r)^{(n-2) / 2}} Z_{m}^{l}(d \lambda) .
$$

Then formula (1) can be rewritten in the following way:

$$
\xi(x)=c_{n} \sum_{m \geq 0} \sum_{l=1}^{h(m, n)} S_{m}^{l}(\bar{\varphi}) \zeta_{m}^{l}(r) .
$$

Moreover, assumptions (2) imply that $\zeta_{m}^{l}(r)$ are uncorrelated random variables for different $m$ and $l$, that is,

$$
\begin{gathered}
\mathrm{E} \zeta_{m}^{l}(r) \overline{\zeta_{p}^{q}(r)}=\delta_{m}^{p} \delta_{l}^{q} b_{m}(r) \\
b_{m}(r)=\int_{0}^{\infty} \frac{J_{m+(n-2) / 2}^{2}(\lambda r)}{(\lambda r)^{n-2}} d \Phi(\lambda)
\end{gathered}
$$

\section{The PROBlem of interpolation of A HOMOGENEOUS AND ISOTROPIC RANDOM FIELD ON $R^{n}$}

Consider the problem of interpolation of a random field $\xi(x)$ at an arbitrary point $y$ belonging to the interior of the sphere $S_{n}$ after observations on the sphere. This problem is reduced to that of finding the projection $\widehat{\xi(y)}$ of the element $\xi(y)$ onto the subspace $H_{\xi}(r)$ obtained by closing, in the mean square sense, of linear combinations of random variables $\{\xi(x):|x|=r\}$. In other words, $\widehat{\xi(y)} \in H_{\xi}(r)=\operatorname{Cl}\left\{\sum \alpha_{k} \xi\left(x_{k}\right),\left|x_{k}\right|=r\right\}$. Then, by Theorem 3 in [2, I, $\S 1]$, the element $\widehat{\xi(y)}$ of the space $H_{\xi}(r)$ can be expanded as follows:

$$
\widehat{\xi(y)}=c_{n} \sum_{m \geq 0} \sum_{l=1}^{h(m, n)} c_{m}^{l} \zeta_{m}^{l}(r)
$$

where $\sum_{m \geq 0} \sum_{l=1}^{h(m, n)}\left|c_{m}^{l}\right|^{2} b_{m}(r)<\infty$.

Moreover, Theorem 1 in [2, IV, §1] solves the above problem of interpolation in the following way:

$$
\widehat{\xi(y)}=c_{n} \sum_{m \geq 0} \sum_{l=1}^{h(m, n)} c_{m}^{l}(y) \zeta_{m}^{l}(r)
$$

where $c_{m}^{l}(y)$ are defined by the formula

$$
c_{m}^{l}(y)=\frac{\int_{0}^{\infty} \frac{J_{m+(n-2) / 2}(\lambda r)}{(\lambda r)^{(n-2) / 2}} \cdot \frac{J_{m+(n-2) / 2}(\lambda \rho)}{(\lambda \rho)^{(n-2) / 2}} d \Phi(\lambda)}{b_{m}(r)} \cdot S_{m}^{l}(\bar{\psi})
$$

and where $(\rho, \bar{\psi})=\left(\rho, \psi_{1}, \ldots, \psi_{n-1}\right)$ are the spherical coordinates of the point $y$.

Let

$$
b_{m}(r, \rho)=\int_{0}^{\infty} \frac{J_{m+(n-2) / 2}(\lambda r)}{(\lambda r)^{(n-2) / 2}} \cdot \frac{J_{m+(n-2) / 2}(\lambda \rho)}{(\lambda \rho)^{(n-2) / 2}} d \Phi(\lambda)
$$


Then formula (9) can be rewritten as follows:

$$
c_{m}^{l}(y)=\frac{b_{m}(r, \rho)}{b_{m}(r)} \cdot S_{m}^{l}(\bar{\psi}) .
$$

Note that interpolation formula (8) involves a sequence of random measures $Z_{m}^{l}$. Practical application of (8) suggests introducing the values of $\xi(x), x \in S_{n}$, which are observed directly, into the formula. This interpolation formula is as follows [2, IV, §1]:

$$
\widehat{\xi(y)}=c(y) \int_{\mathrm{S}_{\mathrm{n}}} \xi(x) d \mu_{n},
$$

where

$$
c(y)=\frac{1}{\omega_{n}} \cdot \frac{b_{0}(r, \rho)}{b_{0}(r)},
$$

$\mu_{n}$ is the Lebesgue measure on the unit sphere $\mathbb{R}^{n}$ and $\omega_{n}$ is the surface area of this sphere.

The corresponding error of interpolation $\sigma^{2}=\mathrm{E}(\xi(y)-\widehat{\xi(y)})^{2}$ is

$$
\sigma^{2}=\varphi(0)-\frac{c_{n}^{2}}{\omega_{n}} \cdot \frac{b_{0}^{2}(r, \rho)}{b_{0}(r)}
$$

(see [2, §4.1]).

\section{Finite Approximation}

In practical applications of formula (12), the integral $\int_{S_{n}} \xi(x) d \mu_{n}$ is approximated by the corresponding integral sum. In other words, the interpolation in the space

$$
H_{\xi}(r)=\mathrm{Cl}\left\{\sum \alpha_{k} \xi\left(x_{k}\right),\left|x_{k}\right|=r\right\}
$$

is replaced by the interpolation of the variable $\xi(y)$ after observations in the space

$$
H_{X}(r)=\left\{\sum \alpha_{k} \xi\left(x_{k}\right), x_{k} \in X\right\},
$$

where $X=\left\{x_{1}, \ldots, x_{N}\right\}$ is a finite set of observations on the sphere $S_{n}$.

Therefore integral (12) is replaced by a finite linear combination (15) of values belonging to the set $X$. This leads to the problem of the accuracy of this substitution and therefore to that of the accuracy of the approximation by interpolation formula (12).

Fix a set $X=\left\{x_{1}, \ldots, x_{N}\right\}$. Since $H_{X} \subset H_{\xi}$, properties of the mean square projection imply the following identity for any $\widehat{\xi_{X}(y)} \in H_{X}$ :

$$
\sigma_{X}^{2} \equiv \mathrm{E}\left(\xi(y)-\widehat{\xi_{X}(y)}\right)^{2}=\sigma^{2}+\widehat{\sigma_{X}^{2}} \equiv \mathrm{E}(\xi(y)-\widehat{\xi(y)})^{2}+\mathrm{E}\left(\widehat{\xi(y)}-\widehat{\xi_{X}(y)}\right)^{2} .
$$

By definition (15), expansion (4) yields the following equality:

$$
\widehat{\xi_{X}(y)}=\sum_{k=1}^{N} \alpha_{k} c_{n} \sum_{m \geq 0} \sum_{l=1}^{h(m, n)} S_{m}^{l}\left(\overline{\varphi_{k}}\right) \zeta_{m}^{l}(r)=c_{n} \sum_{m \geq 0} \sum_{l=1}^{h(m, n)}\left(\sum_{k=1}^{N} \alpha_{k} S_{m}^{l}\left(\overline{\varphi_{k}}\right)\right) \zeta_{m}^{l}(r),
$$

where $\left(r, \overline{\varphi_{k}}\right)=\left(r, \varphi_{k}^{(1)}, \ldots, \varphi_{k}^{(n-1)}\right)$ are the spherical coordinates of $x_{k}$.

Recall that random variables (5) are uncorrelated. Thus we obtain by interpolation formula (8) for $\widehat{\xi(y)}$ and by the addition theorem for the spherical harmonics with $n>2$ 
that

$$
\begin{aligned}
& \widehat{\sigma_{X}^{2}}=\mathrm{E}\left(\widehat{\xi(y)}-\widehat{\xi_{X}(y)}\right)^{2} \\
& =c_{n}^{2} \sum_{m \geq 0} \sum_{l=1}^{h(m, n)}\left|\sum_{k=1}^{N} \alpha_{k} S_{m}^{l}\left(\overline{\varphi_{k}}\right)-\frac{b_{m}(r, \rho)}{b_{m}(r)} S_{m}^{l}(\bar{\psi})\right|^{2} \mathrm{E}\left|\zeta_{m}^{l}(r)\right|^{2} \\
& =c_{n}^{2} \sum_{m \geq 0} b_{m}(r) \sum_{l=1}^{h(m, n)}\left\{\sum_{k, j=1}^{N} \alpha_{k} \alpha_{j} S_{m}^{l}\left(\overline{\varphi_{k}}\right) \overline{S_{m}^{l}\left(\overline{\varphi_{j}}\right)}\right. \\
& -\frac{b_{m}(r, \rho)}{b_{m}(r)}\left(S_{m}^{l}(\bar{\psi}) \overline{\sum_{k=1}^{N} \alpha_{k} S_{m}^{l}\left(\overline{\varphi_{k}}\right)}+\overline{S_{m}^{l}(\bar{\psi})} \sum_{k=1}^{N} \alpha_{k} S_{m}^{l}\left(\overline{\varphi_{k}}\right)\right) \\
& \left.+\frac{b_{m}^{2}(r, \rho)}{b_{m}^{2}(r)}\left|S_{m}^{l}(\bar{\psi})\right|^{2}\right\} \\
& =\frac{c_{n}^{2}}{\omega_{n}} \sum_{m \geq 0} b_{m}(r) h(m, n)\left\{\sum_{k, j=1}^{N} \alpha_{k} \alpha_{j} \frac{C_{m}^{(n-2) / 2}\left(\cos \theta_{k j}\right)}{C_{m}^{(n-2) / 2}(1)}\right. \\
& \left.-2 \frac{b_{m}(r, \rho)}{b_{m}(r)} \sum_{k=1}^{N} \alpha_{k} \frac{C_{m}^{(n-2) / 2}\left(\cos \theta_{k}\right)}{C_{m}^{(n-2) / 2}(1)}+\frac{b_{m}^{2}(r, \rho)}{b_{m}^{2}(r)}\right\},
\end{aligned}
$$

where $\theta_{k j}$ and $\theta_{j}$ are the angles between the vectors $x_{k}$ and $x_{j}$ and $x_{j}$ and $y$, respectively. Here, the $C_{m}^{\nu}(x), m \geq 0$, stand for Gegenbauer polynomials, which can be defined as the coefficients of the following expansion:

$$
\left(1-2 t x+t^{2}\right)^{-\nu}=\sum_{m=0}^{\infty} C_{m}^{\nu}(x) t^{m}
$$

Note that the function on the left-hand side of (18) is the generating function of Gegenbauer polynomials.

Observe that

$$
\frac{h(m, n)}{C_{m}^{(n-2) / 2}(1)}=\frac{(2 m+n-2)(m+n-3) ! m !(n-3) !}{(n-2) ! m !(m+n-3) !}=\frac{(2 m+n-2)}{(n-2)},
$$

$c_{n}^{2} / \omega_{n}=2^{n-2} \Gamma^{2}(n / 2)$, and apply the addition theorem for cylindrical functions to the above result:

$$
\begin{aligned}
\widehat{\sigma_{X}^{2}}=\frac{c_{n}^{2}}{\omega_{n}}\left\{\frac{2}{(n-2)} \sum_{k, j=1}^{N} \alpha_{k} \alpha_{j} \int_{0}^{\infty} \sum_{m \geq 0}\left(m+\frac{n-2}{2}\right)\right. \\
\quad \times \frac{J_{m+(n-2) / 2}^{2}(\lambda r)}{(\lambda r)^{n-2}} C_{m}^{(n-2) / 2}\left(\cos \theta_{k j}\right) d \Phi(\lambda) \\
-\frac{4}{(n-2)} \sum_{j=1}^{N} \alpha_{j} \int_{0}^{\infty} \sum_{m \geq 0}\left(m+\frac{n-2}{2}\right) \frac{J_{m+(n-2) / 2}(\lambda r)}{(\lambda r)^{(n-2) / 2}} \\
\quad \times \frac{J_{m+(n-2) / 2}(\lambda \rho)}{(\lambda \rho)^{(n-2) / 2}} C_{m}^{(n-2) / 2}\left(\cos \theta_{j}\right) d \Phi(\lambda) \\
\left.+\sum_{m \geq 0} h(m, n) \frac{b_{m}^{2}(r, \rho)}{b_{m}(r)}\right\}
\end{aligned}
$$




$$
\begin{aligned}
= & 2^{(n-2) / 2} \Gamma\left(\frac{n}{2}\right) \sum_{k, j=1}^{N} \alpha_{k} \alpha_{j} \int_{0}^{\infty} \frac{J_{(n-2) / 2}\left(\lambda R_{k j}\right)}{\left(\lambda R_{k j}\right)^{(n-2) / 2}} d \Phi(\lambda) \\
& -2^{n / 2} \Gamma\left(\frac{n}{2}\right) \sum_{j=1}^{N} \alpha_{j} \int_{0}^{\infty} \frac{J_{(n-2) / 2}\left(\lambda R_{j}\right)}{\left(\lambda R_{j}\right)^{(n-2) / 2}} d \Phi(\lambda) \\
& +2^{n-2} \Gamma^{2}\left(\frac{n}{2}\right) \sum_{m \geq 0} h(m, n) \frac{b_{m}^{2}(r, \rho)}{b_{m}(r)},
\end{aligned}
$$

where $R_{k j}=2 r \sin \left(\theta_{k j} / 2\right), R_{j}=2 \sqrt{r^{2}+\rho^{2}-2 r \rho \cos \theta_{j}}$, and where $\theta_{k j}$ and $\theta_{j}$ are as defined above.

Define

$$
\begin{gathered}
T_{k j}=2^{(n-2) / 2} \Gamma\left(\frac{n}{2}\right) \int_{0}^{\infty} \frac{J_{(n-2) / 2}\left(\lambda R_{k j}\right)}{\left(\lambda R_{k j}\right)^{(n-2) / 2}} d \Phi(\lambda), \quad k, j=1, \ldots, N, \\
t_{j}=2^{(n-2) / 2} \Gamma\left(\frac{n}{2}\right) \int_{0}^{\infty} \frac{J_{(n-2) / 2}\left(\lambda R_{j}\right)}{\left(\lambda R_{j}\right)^{(n-2) / 2}} d \Phi(\lambda), \quad j=1, \ldots, N, \\
b=2^{n-2} \Gamma^{2}\left(\frac{n}{2}\right) \sum_{m \geq 0} h(m, n) \frac{b_{m}^{2}(r, \rho)}{b_{m}(r)} .
\end{gathered}
$$

Then

$$
\widehat{\sigma_{X}^{2}}=\alpha^{\prime} T \alpha-2 t \alpha+b
$$

Identity (17) implies that the matrix $T=\left(T_{k j} ; k, j=1, \ldots, N\right)$ constituting quadratic form (21) is symmetric and positive definite. Therefore the criterion of minimum of a positive definite quadratic form enables us to obtain the following result.

Theorem 1. Assume that $X=\left\{x_{1}, \ldots, x_{N}\right\}$ is a fixed set of observations on the sphere $S_{n}$, and let $x_{k}=\left(r, \overline{\varphi_{k}}\right)=\left(r, \varphi_{k}^{(1)}, \ldots, \varphi_{k}^{(n-1)}\right)$. The best interpolation, in the sense of the minimum mean square error, of the quantity $\xi(y)$ by variables belonging to $H_{X}(r)$ is given by

$$
\widehat{\xi(y)}=\sum_{k=1}^{N} \alpha_{k} \xi\left(x_{k}\right)
$$

where the vector $\alpha=\left(\alpha_{1}, \ldots, \alpha_{N}\right)$ is a solution of the system

$$
\sum_{k=1}^{N} \alpha_{k} \int_{0}^{\infty} \frac{J_{(n-2) / 2}\left(\lambda R_{k j}\right)}{\left(\lambda R_{k j}\right)^{(n-2) / 2}} d \Phi(\lambda)=\int_{0}^{\infty} \frac{J_{(n-2) / 2}\left(\lambda R_{j}\right)}{\left(\lambda R_{j}\right)^{(n-2) / 2}} d \Phi(\lambda), \quad j=1, \ldots, N
$$

In this case, the mean square error is

$$
\begin{aligned}
\sigma_{X}^{2} \equiv & \mathrm{E}\left(\xi(y)-\widehat{\xi_{X}(y)}\right)^{2}=\sigma^{2}+\widehat{\sigma_{X}^{2}} \\
= & \sigma^{2}+2^{n-2} \Gamma^{2}\left(\frac{n}{2}\right) \sum_{m \geq 0} h(m, n) \frac{b_{m}^{2}(r, \rho)}{b_{m}(r)} \\
& -2^{(n-2) / 2} \Gamma\left(\frac{n}{2}\right) \sum_{j=1}^{N} \alpha_{j} \int_{0}^{\infty} \frac{J_{(n-2) / 2}\left(\lambda R_{j}\right)}{\left(\lambda R_{j}\right)^{(n-2) / 2}} d \Phi(\lambda) .
\end{aligned}
$$

Proof. By (21),

$$
\widehat{\sigma_{X}^{2}}=\alpha^{\prime} T \alpha-2 t \alpha+b
$$


Therefore the necessary and sufficient condition of the minimum takes the form of the following system:

or

$$
\frac{\partial}{\partial \alpha} \widehat{\sigma_{X}^{2}} \equiv 2 T \alpha-2 t=0
$$

$$
0=\sum_{k=1}^{N} \alpha_{k} \int_{0}^{\infty} \frac{J_{(n-2) / 2}\left(\lambda R_{k j}\right)}{\left(\lambda R_{k j}\right)^{(n-2) / 2}} d \Phi(\lambda)-\int_{0}^{\infty} \frac{J_{(n-2) / 2}\left(\lambda R_{j}\right)}{\left(\lambda R_{j}\right)^{(n-2) / 2}} d \Phi(\lambda) .
$$

Then the mean square error equals

$$
\widehat{\sigma_{X}^{2}}=\alpha^{\prime} T \alpha-2 t \alpha+b=t \alpha-2 t \alpha+b=b-t \alpha
$$

as is required to prove.

Example 4.1. Consider the problem of interpolation at the center of a circle in the plane, that is, take the case where $y=0$ and $n=2$. Assume that the points of the set $X_{N}=\left\{x_{1}, \ldots, x_{N}\right\}$ are uniformly distributed on the circumference $S_{2}$, that is,

$$
\varphi_{k}=2 \pi(k-1) / N, \quad 1 \leq k \leq N .
$$

Suppose that the spectral measure is concentrated at a single point, that is,

$$
\Phi(\lambda)= \begin{cases}1, & \lambda=1 \\ 0, & \lambda \neq 1\end{cases}
$$

System (22) can be rewritten in matrix form as follows:

$$
A \alpha=b,
$$

where, by the above assumptions,

$$
A_{i j}=J_{0}\left(2 r \sin \left(\frac{\varphi_{i}-\varphi_{j}}{2}\right)\right) \quad \text { and } \quad b_{j}=J_{0}(r) .
$$

By the definition of the Bessel function, the diagonal entries of the matrix $A$ are all equal to one:

$$
A_{i i}=J_{0}\left(2 r \sin \left(\frac{\varphi_{i}-\varphi_{i}}{2}\right)\right)=J_{0}(2 r \cdot 0)=1 .
$$

It is clear that $A_{i j}=A_{j i}$. Therefore the matrix $A$ is symmetric. Moreover, one can readily see that $\ldots=A_{i-1, j-1}=A_{i j}=A_{i+1, j+1}=A_{i+2, j+2}$, and so on. Since the points of the set $X_{N}=\left\{x_{1}, \ldots, x_{N}\right\}$ are uniformly distributed, we obtain

$$
A_{1 j}=A_{1, N-2+j} \text {. }
$$

Hence the matrix $A$ is as follows:

$$
\left(\begin{array}{cccccc}
1 & A_{12} & A_{13} & \ldots & A_{13} & A_{12} \\
A_{12} & 1 & A_{12} & A_{13} & \ldots & A_{13} \\
A_{13} & A_{12} & 1 & A_{12} & \ldots & A_{14} \\
\vdots & \vdots & \vdots & \ddots & \vdots & \vdots \\
A_{13} & A_{14} & \ldots & A_{12} & 1 & A_{12} \\
A_{12} & A_{13} & A_{14} & \ldots & A_{12} & 1
\end{array}\right) .
$$

It is easy to observe that each subsequent row of this matrix is obtained by cyclic permutation of the previous one. Therefore the entries of the first row determine the whole matrix. The sum of entries in each row is constant:

$$
a:=\sum_{j=1}^{N} A_{i j}=\sum_{j=1}^{N} A_{1 j}, \quad i=1, \ldots, N .
$$


This readily implies that the vector $\alpha^{\prime}=a^{-1}\left(J_{0}(r), J_{0}(r), \ldots, J_{0}(r)\right)$ is a solution of system (25). The corresponding mean square error is

$$
\sigma_{X}^{2}=\sigma^{2}+\widehat{\sigma_{X}^{2}}=\sigma^{2}+1-N \frac{J_{0}^{2}(r)}{a} .
$$

\section{A LiMIT THEOREM FOR THE ERROR OF INTERPOLATION}

In the case where finding an exact solution of system of equations (22) is troublesome, one can use an approximation.

Denote by

$$
F_{N}(\bar{\varphi})=\sum_{k: \overline{\varphi_{k}} \leq \bar{\varphi}} \alpha_{k}, \quad \varphi_{1} \in[0,2 \pi], \quad \varphi_{i} \in[0, \pi], i=2, \ldots, n-1,
$$

the cumulative distribution function of the coefficients $\left\{\alpha_{1}, \ldots, \alpha_{N}\right\}$. Here, the inequality $\overline{\varphi_{k}} \leq \bar{\varphi}$ stands for the system of the coordinatewise inequalities $\varphi_{k}^{(i)} \leq \varphi_{i}, i=1, \ldots, n-1$.

By (17), we write

$$
\begin{aligned}
& \widehat{\sigma_{X}^{2}}=c_{n}^{2} \sum_{m \geq 0} b_{m}(r) \sum_{l=1}^{h(m, n)}\left\{\int_{\Pi_{n}} \int_{\Pi_{n}} S_{m}^{l}(\bar{\varphi}) \overline{S_{m}^{l}(\bar{\theta})} d F_{N}(\bar{\varphi}) d F_{N}(\bar{\theta})\right. \\
&-\frac{b_{m}(r, \rho)}{b_{m}(r)}\left(S_{m}^{l}(\bar{\psi}) \int_{\Pi_{n}} \overline{S_{m}^{l}(\bar{\varphi})} d F_{N}(\bar{\varphi})\right.\left.\left.\overline{S_{m}^{l}(\bar{\psi})} \int_{\Pi_{n}} S_{m}^{l}(\bar{\varphi}) d F_{N}(\bar{\varphi})\right)+\frac{b_{m}^{2}(r, \rho)}{b_{m}^{2}(r)}\left|S_{m}^{l}(\bar{\psi})\right|^{2}\right\} \\
&=c_{n}^{2} \sum_{m \geq 0} b_{m}(r) \sum_{l=1}^{h(m, n)}\left\{\left|\int_{\Pi_{n}} S_{m}^{l}(\bar{\varphi}) d F_{N}(\bar{\varphi})\right|^{2}\right.-\frac{b_{m}(r, \rho)}{b_{m}(r)}\left(S_{m}^{l}(\bar{\psi}) \int_{\Pi_{n}} \overline{S_{m}^{l}(\bar{\varphi})} d F_{N}(\bar{\varphi})\right. \\
&\left.\left.+\overline{S_{m}^{l}(\bar{\psi})} \int_{\Pi_{n}} S_{m}^{l}(\bar{\varphi}) d F_{N}(\bar{\varphi})\right)+\frac{b_{m}^{2}(r, \rho)}{b_{m}^{2}(r)}\left|S_{m}^{l}(\bar{\psi})\right|^{2}\right\} \\
&=c_{n}^{2} \sum_{m \geq 0} b_{m}(r) \sum_{l=1}^{h(m, n)}\left|\int_{\Pi_{n}} S_{m}^{l}(\bar{\varphi}) d F_{N}(\bar{\varphi})-\frac{b_{m}(r, \rho)}{b_{m}(r)} S_{m}^{l}(\bar{\psi})\right|^{2}
\end{aligned}
$$

where $\Pi_{n}=[0,2 \pi] \times \underbrace{[0, \pi] \times \cdots \times[0, \pi]}_{n-2}$.

Theorem 2. Assume that the sequence of functions $F_{N}(\cdot)$ on $\Pi_{n}$ having uniformly bounded variations converges weakly to a function $F(\cdot)$ with bounded variation. Then the limit mean square error of approximation of the integral $\widehat{\xi(y)}$ is equal to

$$
\widehat{\sigma_{\infty}^{2}}=c_{n}^{2} \sum_{m \geq 0} b_{m}(r) \sum_{l=1}^{h(m, n)}\left|\int_{\Pi_{n}} S_{m}^{l}(\bar{\varphi}) d F(\bar{\varphi})-c_{m}^{l}(y)\right|^{2},
$$

where $c_{m}^{l}(y)=S_{m}^{l}(\bar{\psi}) b_{m}(r, \rho) / b_{m}(r)$.

Proof. By continuity of Bessel functions and by the definition of the convergence of functions with bounded variations, taking the limit as $N \rightarrow \infty$ in (28) gives the required result. 
Corollary 1. Under the assumptions of Theorem 2, the asymptotic mean square error of approximation equals zero if and only if

$$
\int_{\Pi_{n}} S_{m}^{l}(\bar{\varphi}) d F(\bar{\varphi})=c_{m}^{l}(y)
$$

for all $m \geq 0$ and $l=1, \ldots, h(m, n)$.

Corollary 2. If

$$
\sum_{m \geq 0} \sum_{l=1}^{h(m, n)}\left|c_{m}^{l}(y)\right|^{2}<+\infty,
$$

then the asymptotic error equals zero if and only if the limit function satisfies

$$
d F(\bar{\varphi})=C(y, x) d \bar{\varphi}
$$

for $\bar{\varphi} \in \Pi_{n}$, where

$$
C(y, x)=\frac{1}{\omega_{n}(n-2)} \sum_{m \geq 0} \frac{b_{m}(r, \rho)}{b_{m}(r)}(2 m+n-2) C_{m}^{(n-2) / 2}(\cos \theta),
$$

$\theta$ is the angle between the vectors $x$ and $y$, and where the $C_{m}^{\nu}(x), m \geq 0$, are the Gegenbauer polynomials defined by formula (18).

Proof. Observe that the integrals $\int_{\Pi_{n}} S_{m}^{l}(\bar{\varphi}) d F(\bar{\varphi})$ are generalized Fourier coefficients of the function $d F(\bar{\varphi})$ with respect to the system of orthonormal functions

$$
\left\{S_{m}^{l}(\bar{\varphi})\right\}_{m \geq 0, l=1, \ldots, h(m, n)} .
$$

Then, by the addition theorem for spherical harmonics, we obtain from Corollary 1 and condition (31)

$$
\begin{aligned}
\frac{d F(\bar{\varphi})}{d \bar{\varphi}} & =\sum_{m \geq 0} \sum_{l=1}^{h(m, n)} \frac{b_{m}(r, \rho)}{b_{m}(r)} S_{m}^{l}(\bar{\psi}) S_{m}^{l}(\bar{\varphi}) \\
& =\sum_{m \geq 0} \frac{b_{m}(r, \rho)}{b_{m}(r)} \frac{h(m, n)}{\omega_{n}} \frac{C_{m}^{(n-2) / 2}(\cos \theta)}{C_{m}^{(n-2) / 2}(1)} \\
& =\frac{1}{\omega_{n}} \sum_{m \geq 0} \frac{b_{m}(r, \rho)}{b_{m}(r)} \frac{(2 m+n-2)(m+n-3) ! m !(n-3) !}{(n-2) ! m !(m+n-3) !} C_{m}^{(n-2) / 2}(\cos \theta) \\
& =C(y, x) .
\end{aligned}
$$

Corollary 3. Let $X_{N}=\left\{x_{1}, \ldots, x_{N}\right\}$ be an arbitrary sequence of sets where $x_{k}=\left(r, \overline{\varphi_{k}}\right)$, $0<\varphi_{1}^{(1)}<\varphi_{2}^{(1)}<\cdots<\varphi_{N}^{(1)}=2 \pi$, and $0<\varphi_{1}^{(i)}<\varphi_{2}^{(i)}<\cdots<\varphi_{N}^{(i)}=\pi, i=2, \ldots, n-1$. If condition (31) holds and the coefficients

$$
\alpha_{k}=C\left(y, x_{k}\right) \prod_{i=1}^{n-1}\left(\varphi_{k}^{(i)}-\varphi_{k-1}^{(i)}\right)
$$

are such that $\max \left|\prod_{i=1}^{n-1}\left(\varphi_{k}^{(i)}-\varphi_{k-1}^{(i)}\right)\right| \rightarrow 0$ as $N \rightarrow \infty$, then the mean square error of approximation $\widehat{\sigma_{X}^{2}}$ tends to zero as $N \rightarrow \infty$.

Proof. On one hand, it follows from (17) that the mean square error of approximation equals

$$
\widehat{\sigma_{X}^{2}}=c_{n}^{2} \sum_{m \geq 0} b_{m}(r) \sum_{l=1}^{h(m, n)}\left|\int_{\Pi_{n}} S_{m}^{l}(\bar{\varphi}) d F_{N}(\bar{\varphi})-c_{m}^{l}(y)\right|^{2} .
$$


On the other hand, we have

$$
\widehat{\sigma_{X}^{2}}=c_{n}^{2} \sum_{m \geq 0} b_{m}(r) \sum_{l=1}^{h(m, n)}\left|\sum_{k=1}^{N} \alpha_{k} S_{m}^{l}\left(\overline{\varphi_{k}}\right)-c_{m}^{l}(y)\right|^{2} .
$$

Substitute the values of $\alpha_{k}$ into the second identity and denote

$$
\Delta \overline{\varphi_{k}}:=\prod_{i=1}^{n-1}\left(\varphi_{k}^{(i)}-\varphi_{k-1}^{(i)}\right) .
$$

Then

$$
\begin{aligned}
& c_{n}^{2} \sum_{m \geq 0} b_{m}(r) \sum_{l=1}^{h(m, n)}\left|\int_{\Pi_{n}} S_{m}^{l}(\bar{\varphi}) d F_{N}(\bar{\varphi})-c_{m}^{l}(y)\right|^{2} \\
& \quad=c_{n}^{2} \sum_{m \geq 0} b_{m}(r) \sum_{l=1}^{h(m, n)}\left|\sum_{k=1}^{N} C\left(y, x_{k}\right) S_{m}^{l}\left(\overline{\varphi_{k}}\right) \Delta \overline{\varphi_{k}}-c_{m}^{l}(y)\right|^{2} .
\end{aligned}
$$

Let $N \rightarrow \infty$ on both sides of the equality. Then we obtain for all $l=1, \ldots, h(m, n)$ and $m \geq 0$ that

$$
\int_{\Pi_{n}} S_{m}^{l}(\bar{\varphi}) d F(\bar{\varphi})=\int_{\Pi_{n}} C(y, x) S_{m}^{l}(\bar{\varphi}) d \varphi
$$

Since $\left\{S_{m}^{l}(\cdot), l=1, \ldots, h(m, n), m \geq 0\right\}$ is a complete system of functions on the sphere $S_{n}$, we have

$$
d F(\bar{\varphi}) \equiv C(y, x) d \varphi .
$$

Corollary 2 implies the required result.

\section{Concluding Remarks}

The problem of interpolation of a random field often occurs in the natural sciences and engineering. In practice, the number of observations is finite and therefore the problem of approximation of a certain interpolation formula arises. A finite approximation of this type is a result of this paper. We have also found an explicit formula for the corresponding mean square error of approximation. The asymptotic behavior of the error is studied given that the number of observation points increases, and conditions of error-free approximation are established. As a result, an optimal location of observation points is recommended.

\section{BIBLIOGRAPHY}

1. M. V. Kartashov, Finite-dimensional interpolation of a random field on the sphere, Teor. Ǐmovir. Mat. Stat. 51 (1995), 53-61; English transl. in Theory Probab. Math. Statist. 51 (1996), 53-61. MR1445052 (97k:60142)

2. M. I. Yadrenko, Spectral Theory of Random Fields, "Vyshcha shkola", Kiev, 1980; English transl., Optimization Software, New York, 1983. MR697386 (84f:60003)

3. N. N. Lebedev, Special Functions and Their Applications, "Nauka", Moscow, 1963; English transl., Dover Publications, Inc., New York, 1972. MR.0350075 (50:2568)

Department of Probability Theory and Mathematical Statistics, Faculty for Mechanics and Mathematics, National Taras Shevchenko University, Volodymyrs'ka Street, 64, Kyiv 01033, UKRAINE

E-mail address: semenovsky@voliacable.com

Received 28/MAR/2005

Translated by V. ZAYATS 